\title{
Determination of refractive index, thickness, and the optical losses of thin films from prism-film coupling measurements
}

\author{
Julien Cardin ${ }^{1, *}$ and Dominique Leduc ${ }^{2}$ \\ 'SIFCOM, CNRS UMR 6176, ENSICAEN, 6 Boulevard du Maréchal-Juin, F-14050 Caen Cedex, France \\ ${ }^{2}$ Université de Nantes, Nantes Atlantique Universités, IREENA, EA1770, Faculté des Sciences et des Techniques, \\ 2 rue de la Houssinière-BP 9208, Nantes F-44000, France \\ ${ }^{*}$ Corresponding author: julien.cardin @ ensicaen.fr
}

Received 4 September 2007; revised 17 December 2007; accepted 3 January 2008; posted 11 January 2008 (Doc. ID 87215); published 28 February 2008

\begin{abstract}
We present a method of analysis of prism-film coupler spectroscopy based on the use of transfer matrix and genetic algorithm, which allows the simultaneous determination of refractive index, thickness, and optical losses of the measured layer. (c) 2008 Optical Society of America

OCIS codes: $\quad 130.3130,120.3940,160.4760,300.1030$.
\end{abstract}

\section{Introduction}

Transparent thin films find wide applications in optics: coating, sensors, integrated components for telecommunications, solar cell, etc. For all these applications, it is necessary to perform an accurate characterization of the optical properties, which include refractive index and optical losses. The optical losses of a thin film have three different origins: surface scattering due to the surface roughness, volume scattering due to volume defects such as inhomogeneity, and volume absorption due to intrinsic absorption [1,2]. From a practical point of view, this characteristic is of particular importance since it can severely degrade or modify the performances of a component.

Several techniques exist which allow the characterization of the optical parameters of thin films. For example, the measurement of reflection and transmission coefficients [3-6] and the spectroscopic ellipsometry [7-9] lead to refractive index, thickness, and extinction factor measurement.

On the other hand, since the 1970 s, a prism-film coupler based method, also called $m$-lines spectro-

0003-6935/08/070894-07\$15.00/0

(C) 2008 Optical Society of America scopy has been developed to characterize thin films. Full-accuracy index and thickness measurements are obtained, for nonbirefringent films [10] and in the absence of nonlinear effects [11], once film thickness exceeds a certain minimum threshold value (typically $300 \mathrm{~nm}$, depending on the film/substrate type) $[12,13]$. This technique is based on a selective excitation, through an evanescent field, of guided modes of a film. The total reflection at the upper boundary of the film is frustrated by the presence of the prism and allows the light to go back into the prism. Since this reflected light carries information on the film, it can be used to determine the parameters of the film. Classically, this method was restricted to the determination of the thickness and the refractive index of the films. The scope of this paper is to present a method of analysis, which allows retrieving the optical losses also from classical prism-film coupler measurements, for films of negligible roughness.

\section{Prism-Film Coupler Angular Reflectivity}

In $m$-lines devices, the sample is pressed against a face of a prism in such a way that it is separated from the prism by a thin air gap with a thickness about a quarter of wavelength of the measurement light. The prism and the film are mounted onto a rotating stage 
that varies the incidence angle. The incoming light is refracted inside the prism and reaches the interface between the prism and the sample. As the refractive index of the prism is higher than that of the sample, for a given range of incidence angles, the light is totally reflected by this interface and then emerges from the prism. However, for some angles, called "synchronous angles," a part of the light is coupled in the waveguide. Depending on refractive indices of layer and cladding, one obtains guided or leaky modes. For both cases, the light coupled inside the film is subtracted to the detected light. Thus, a typical $m$-lines spectrum is made of several absorptionlike peaks, centered around the synchronous angles.

In classical analysis, the propagation constants of the guided modes are deduced from the positions of the synchronous angles, and then the refractive index and thickness are determined by solving the modal dispersion equations. A previous paper [14] on the analysis of $m$-lines spectroscopy with genetic algorithm was also done in the framework of the modal dispersion equations with the synchronous angles as input data. In our case, we are performing a fit on the whole recorded spectrum by using the transfer matrix theory to simulate the angular reflectivity of the prism-film coupler.

Consider the schematic of the $m$-lines prism coupler in Fig. 1. In our model, the prism positioned on the top part of the drawing is considered as a nonabsorbing medium. The optical film is surrounded by a thin air gap and a semi-infinite substrate. The complex refractive indexes, $\tilde{n}=n+i k$, including extinction factor $k$, are used for each layer. We cannot separate the contributions of surface, volume scattering, and volume absorption only from the $m$-lines spectrum. Thus, in our case we consider this extinction factor as an effective extinction factor, which represents a priori the three types of contribution to optical losses. The extinction factors of the air and of the prism were assumed to be zero.

A transfer matrix $M_{j}$, which binds the electromagnetic fields at the backplane of the layer to the fields at its front plane, is associated with both film and air layers $[\underline{15}, \underline{16}]$ :

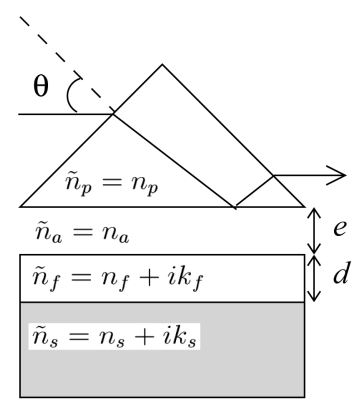

(a)

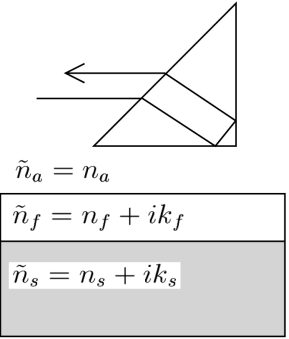

(b)
Fig. 1. Prism coupler configuration: (a) transmission configuration, and (b) reflection configuration.

$$
M_{j}=\left(\begin{array}{cc}
\cos \phi_{j} & -i \gamma_{j}^{-1} \sin \phi_{j} \\
-i \gamma_{j} \sin \phi_{j} & \cos \phi_{j}
\end{array}\right),
$$

with phase factor $\phi_{j}=d_{j}\left[\left(\tilde{n}_{j} k_{0}\right)^{2}-\beta^{2}\right]^{1 / 2}$, where $d_{j}$ is the thickness of the $j$ th layer, $k_{0}$ is the wave vector, and $\beta$ is the propagation constant in the direction parallel to the interface. The transversal component in the direction perpendicular to the interface of the wave vector is $\gamma_{j}=\left(d_{j} k_{0}\right)^{-1} \phi_{j}$ for TE waves and $\gamma_{j}=$ $k_{0} \tilde{n}_{j}^{2} \phi_{j}^{-1}$ for TM waves.

The global transfer matrix $M$ of the system formed by the two layer stack (air gap + thin film) is the product of the individual transfer matrices: $M=$ $M_{a} \cdot M_{f}$ and the power reflection coefficient $R(\theta)$ of the stack surrounded by the prism and the substrate is given by

$$
R(\theta)=\left|\frac{\left(m_{11}+m_{12} \gamma_{s}\right) \gamma_{p}-m_{21}-m_{22} \gamma_{s}}{\left(m_{11}+m_{12} \gamma_{s}\right) \gamma_{p}+m_{21}+m_{22} \gamma_{s}}\right|^{2},
$$

where $\theta$ is the incidence angle of the incoming wave, $m_{i j}$ are the elements of $M$, and $\gamma_{s}$ and $\gamma_{p}$ are, respectively, the transversal component of the wave vector $\left(\gamma_{j}\right.$ factor) in the substrate and in the prism.

Measurements were performed with two different kinds of $m$-lines setups at $632.8 \mathrm{~nm}$ and in TE mode: one commercial setup from Metricon Corporation [17] in transmission configuration (see Fig. 1(a)) and one homemade setup in reflection configuration [18] (see Fig. 1(b)). However, since there is a total internal reflection on both faces of the prism in the latter device (see Fig. 1(b)), the two cases are identical. The incident wave is first refracted inside the prism, then reflected by the two layer stack, and finally refracted in the air before reaching the detector. Hence, the global reflectivity of the prism/film coupler is $T(\theta)_{a / p} R(\theta) T(\theta)_{p / a}$, where $T(\theta)_{a / p}$ and $T(\theta)_{p / a}$ are the classical transmittivity of the prism/air diopter:

$$
\left\{\begin{array}{l}
T(\theta)_{a / p}=\frac{\sqrt{n_{p}^{2}-n_{a}^{2} \sin ^{2} \theta}}{n_{a} \cos \theta}\left|t_{\mathrm{ap}}\right|^{2} \\
T(\theta)_{p / a}=\frac{n_{a} \cos \theta}{\sqrt{n_{p}^{2}-n_{a}^{2} \sin ^{2} \theta}}\left|t_{\mathrm{pa}}\right|^{2},
\end{array}\right.
$$

where $t_{\mathrm{ap}}$ (respectively, $t_{\mathrm{pa}}$ ) is the Fresnel transmission coefficient [19] of the air/prism diopter (respectively, prism/air), and $n_{p}$ and $n_{a}$ are the refractive index of prism and air.

To analyze real measurements, it is necessary to add a transfer function of the apparatus $H(\theta)$ to the calculated reflectivity:

$$
R_{\text {calc }}(\theta)=H(\theta) T(\theta)_{a / p} R(\theta) T(\theta)_{p / a} .
$$

This transfer function contains all the variations of the detected intensity related to the measurement device; it is the ratio of the theoretical and experimental reflectivity without the loaded sample. $H(\theta)$ is determined by measuring the angular 
reflectivity $R_{0}^{\text {meas }}(\theta)$ of the $m$-lines device when no sample is pressed against the prism. The theoretical angular reflectivity without sample $R_{0}^{\text {calc }}(\theta)$ is equal to $T(\theta)_{a / p} T(\theta)_{p / a}$. The transfer function is then $H(\theta)=R_{0}^{\text {calc }}(\theta) / R_{0}^{\text {meas }}(\theta)$. An example of transfer function and of a raw measured reflectivity and a reflectivity corrected by the transfer function is presented in Figs. 2(a) and 2(b).

\section{Fitting by Genetic Algorithm of the Prism-Film Coupler Angular Reflectivity}

Once the theoretical reflection function is calculated, one has to use a numerical procedure to adjust this function to the measured reflectivity. The free parameters of the model are the refractive index $n$, the extinction factor $k$, the thickness $d$ of the film, and the thickness $e$ of the air gap. The genetic algorithms are very well suited to solve this problem since they perform a random search in the parameters space with a weak dependency on initial values and their ability to find global minima. This is a key feature because most of the time one has very imprecise initial guesses on the thickness of the air gap and on the extinction coefficient.

Our fitting process uses a real coded genetic algorithm written by Yedder [20] based on a limited number of classical and efficient well-established operators for genetic algorithms [21]. Let us give an outline of its principle. First the searching space is framed for each parameter; bounds are arbitrarily chosen so that the parameters remain inside physically acceptable limits. To accelerate the convergence of the algorithm, it is possible to determine approximate values $\left\{n_{0}, d_{0}\right\}$ of the refractive index and the thickness of the film by solving the modal dispersion equation and by defining a narrower searching range for these parameters centered around $n_{0}$ and $d_{0}$. Once the searching space is defined, the algorithm works in three steps:

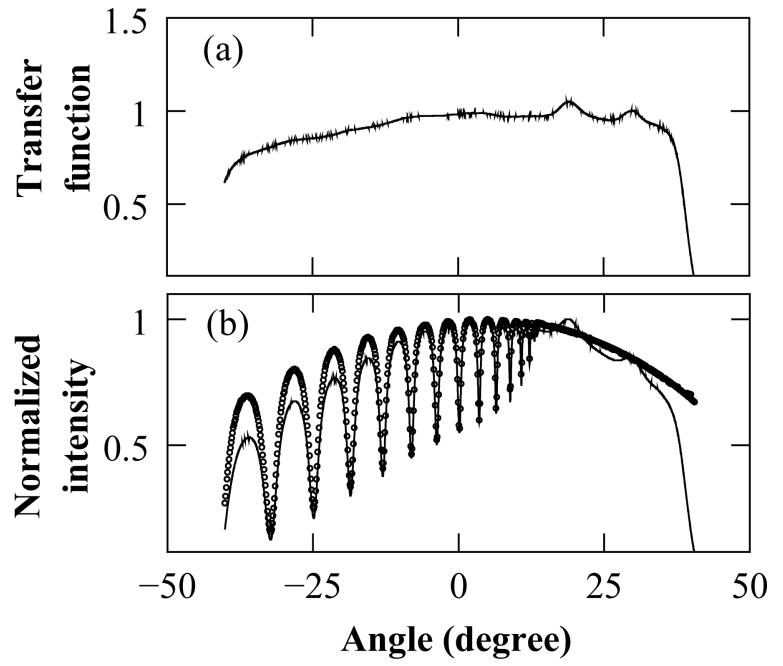

Fig. 2. (a) Transfer function of the Metricon prism-coupler, and (b) $m$-lines spectrum (TE mode) of a photoresist S1818 film: raw data (-) and corrected spectrum $(\mathrm{O})$.
1. A starting population of two layers (thin film +air gap) is initialized; a population size of 10 is randomly created in the framed searching space.

2. An evaluation of the starting population is performed, the reflectivity $R_{\text {calc }}$ of each individual is calculated, and its similarity to the measured reflectivity $R_{\text {meas }}$ is evaluated with a least square estimator:

$$
\sigma_{m}^{2}=\sum_{i=1}^{N} N^{-1}\left[R_{\text {calc }}\left(\theta_{i}\right)-R_{\text {meas }}\left(\theta_{i}\right)\right]^{2}
$$

where $N$ is the number of samples in the measured spectrum. The smallest is $\sigma_{m}$, the best is the individual, so this sigma is taken as the fitness of the fit.

3. A new population is created from the previous offspring by the following operators:

- Selection: a part of the best individuals are chosen, but some individuals with bad performance (i.e., high value of $\sigma_{m}$ ) can be kept; the selection is made by stochastic roulette $[22,23]$.

- An elitism function was used to choose the best of two consecutive generations [24].

- A niching function was used to promote the full searching range exploration [25].

- A sigma scaling technique was used to avoid the selection of a single very good solution and consequently convergence of the algorithm was too rapid [26].

- A mutation operator, which is from a nonuniform type, and with a "simulated binary crossover" (SBX) type crossing [27].

Steps 2 and 3 are iterated until the fitness $\sigma_{m}$ becomes smaller than a given value, and the maximum number of offspring is limited to 50,000 to limit the calculation time. A detailed description of all these procedures can be found in [20].

To test the efficiency of the method, numerical tests were realized. A score of $m$-lines spectra were simulated using the transfer matrix method by varying each parameter one after another around the configuration $n=2.15, k=0.005, d=1.25 \mu \mathrm{m}$, and $e=125 \mathrm{~nm}$. The refractive indexes of the prism and the substrate were, respectively, equal to 2.9 and 1.5. The simulated spectra were used as input data for the genetic algorithm. The program was stopped as soon as $\sigma_{m}$ became smaller than $10^{-4}$. The relative differences between the values obtained with the genetic algorithm and the theoretical one are summarized in Table 1.

A good agreement was found between the initial starting values of simulation and the results given by the genetic algorithm program. The parameters $n$ and $d$ are better reconstructed than the air gap thickness $e$ and the extinction factor $k$, because they have a dominating influence on the shape of the angular reflectivity $R_{\text {calc }}(\theta)$. The errors on these parameters, respectively, $\Delta n$ and $\Delta d$, are of the same 
Table 1. Errors on Paramaters Retrieved by the Genetic Algorithm for the Simulated Spectra; Subscript th Denotes the Theoretical Values

\begin{tabular}{|c|c|c|c|c|c|}
\hline & $\begin{array}{c}\Delta n \\
\left(\times 10^{-4}\right)\end{array}$ & $\begin{array}{c}\Delta k \\
\left(\times 10^{-4}\right)\end{array}$ & $\begin{array}{c}\Delta d \\
(\mathrm{~nm})\end{array}$ & $\begin{array}{c}\Delta e \\
(\mathrm{~nm})\end{array}$ & $\begin{array}{c}\sigma_{m} \\
\left(\times 10^{-5}\right)\end{array}$ \\
\hline \multicolumn{6}{|l|}{$n_{\text {th }}$} \\
\hline 1.900 & 0.9 & 0.1 & 0.1 & 0.1 & 2.8 \\
\hline 2.025 & 0.9 & 0.1 & 0.1 & 0.0 & 3.5 \\
\hline 2.150 & 1.1 & 0.3 & 0.2 & 0.3 & 9.0 \\
\hline 2.275 & 0.7 & 0.6 & 0.1 & 0.0 & 7.0 \\
\hline 2.400 & 0.2 & 0.0 & 0.0 & 0.2 & 6.0 \\
\hline \multicolumn{6}{|l|}{$k_{\text {th }}$} \\
\hline 0.0010 & 0.0 & 0.1 & 0.0 & 0.3 & 6.2 \\
\hline 0.0125 & 4.2 & 4.1 & 0.7 & 0.4 & 9.9 \\
\hline 0.0250 & 25.8 & 1.3 & 4.3 & 0.2 & 9.5 \\
\hline 0.0370 & 19.3 & 4.1 & 2.8 & 0.0 & 1.1 \\
\hline 0.0500 & 53.9 & 5.1 & 8.5 & 0.4 & 8.8 \\
\hline \multicolumn{6}{|l|}{$d_{\mathrm{th}}$} \\
\hline 0.500 & 0.6 & 0.2 & 0.0 & 0.3 & 6.0 \\
\hline 0.875 & 1.5 & 0.1 & 0.2 & 0.2 & 5.8 \\
\hline 1.250 & 1.7 & 1.1 & 0.2 & 0.2 & 7.0 \\
\hline 1.625 & 2.8 & 0.6 & 0.6 & 0.2 & 6.3 \\
\hline 2.000 & 3.1 & 0.2 & 0.9 & 0.0 & 8.1 \\
\hline \multicolumn{6}{|l|}{$e_{\text {th }}$} \\
\hline 50.00 & 0.2 & 0.0 & 0.0 & 0.0 & 9.0 \\
\hline 87.50 & 0.2 & 0.1 & 0.0 & 0.1 & 9.0 \\
\hline 125.00 & 0.4 & 0.3 & 0.1 & 0.1 & 9.0 \\
\hline 162.50 & 0.3 & 0.3 & 0.2 & 0.3 & 2.0 \\
\hline 200.00 & 0.5 & 0.6 & 0.1 & 0.2 & 3.0 \\
\hline
\end{tabular}

order as $\sigma_{m} ; \Delta n$ remains smaller than $10^{-4}$ and $\Delta d$ smaller than $1 \mathrm{~nm}$. However, for $k>0.025: \Delta n$ reaches $5.410^{-3}$ and $\Delta d$ is about $9 \mathrm{~nm}$. This can be explained by the fact that spectra become flatter when the $k$ values increase. The reconstructed $k$ and $e$ are less accurate; however the error on $e$ remains smaller than $0.5 \%$ in relative value, and the error on $k$ is generally smaller than $0.5 \%$ and never exceeds $3.3 \%$. These results demonstrate that the genetic algorithm is able to retrieve the characteristics of a thin film with a remarkable accuracy.

\section{Results and Discussion}

To validate our procedure experimentally, we measured the $m$-lines spectrum of different samples: films of photoresist S1818 [28] spin coated on silicon substrates $\left(\tilde{n}_{\text {silicon }}=3.91 \overline{90}+i 0.0123[29]\right)$, films of $\mathrm{ZnO}: \mathrm{Al}$ deposited by $\mathrm{rf}$ magnetron sputtering on glass substrates Corning $1737 \mathrm{~F} \quad\left(\tilde{n}_{1737 \mathrm{~F}}=\right.$ $1.5172+i 0.0$ ), and an indium tin oxide (ITO) layer on glass substrate $\left(\tilde{n}_{\text {glass }}=1.5157+i 0.0\right)$ from Präzisions Glas \& Optik GmbH [30]. Atomic force microscope (AFM) measurements showed that the samples have a small roughness $(\leq 4 \mathrm{~nm})$ and no correlation length at the scale of the wavelength of measurement. Using the theory of light scattering by rough surface developed by Bennet and Porteus [31] and Davies [32] and taking into account our experimental roughness we have calculated that the total scattered light by surface is $<1 \%$ of total reflected light. We conclude that the losses due to surface scattering can then be neglected in the following given results.

The measured spectra and the fits obtained with the genetic algorithm are shown in Figs. 4-6. The reconstructed parameters with uncertainties in confidence limits of $95 \%$ are summarized in Tables 2 and 3 . Because of the fitting procedure with the genetic algorithm, a classical certainty calculation by differentiation of a modal equation was not possible. The certainty analysis was performed by Monte Carlo simulations. First the experimental noise distribution (Fig. 3) was measured. This distribution follows a normal law with a standard deviation of $1 \%$. Then, for each measurement, a Gaussian noise with standard deviation corresponding to the experimental one was added to several hundreds of spectra simulated with parameters given by the fit. All noisy spectra were fitted with the genetic algorithm leading to the distributions of values of the parameters. As these distributions followed normal laws, we used twice the standard deviation (confidence interval of 95\%) as the uncertainty for each parameter.

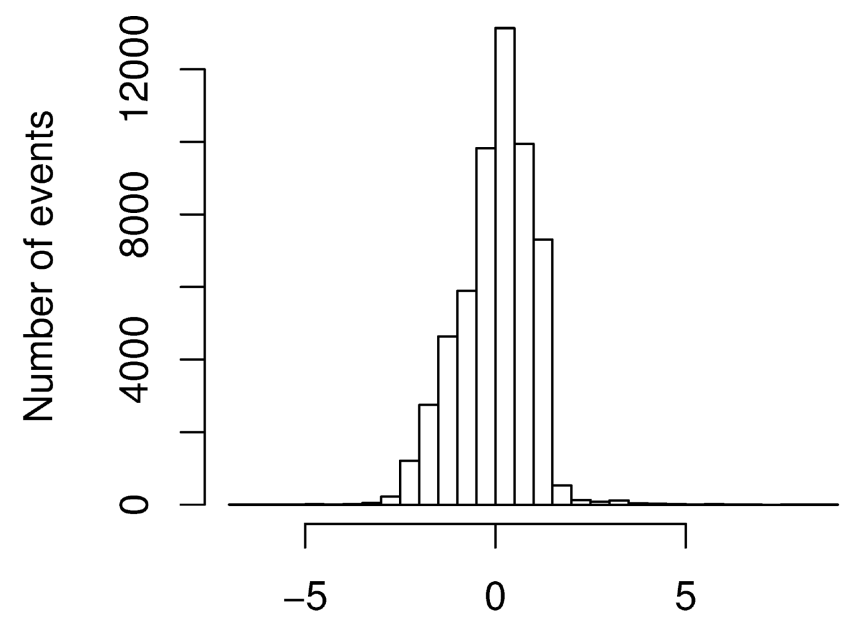

Relative deviation from the mean (\%)

Fig. 3. Distribution of experimental noise.

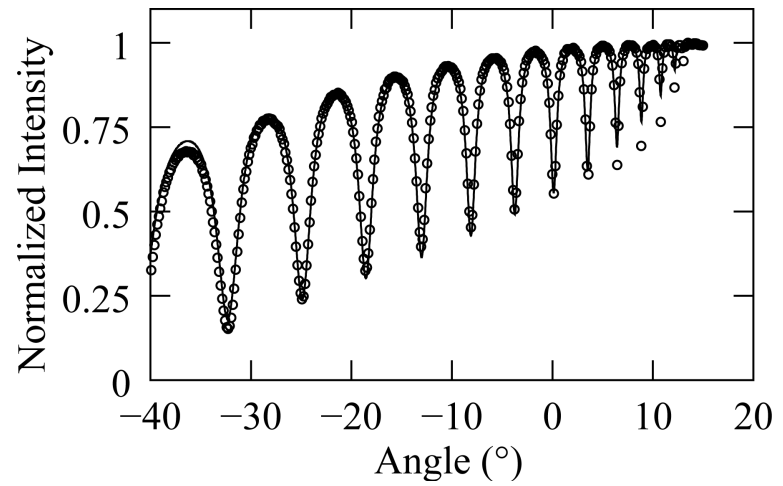

Fig. 4. Measured (O) and fitted (-) $m$-lines spectra of an S1818 film on silicon in TE mode. 


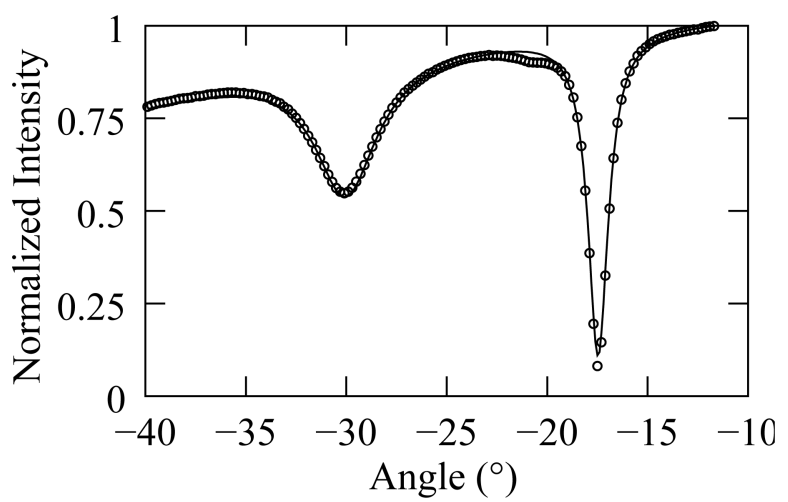

Fig. 5. Measured (O) and fitted (-) $m$-lines spectra of different $\mathrm{ZnO}$ film on glass in TE mode.

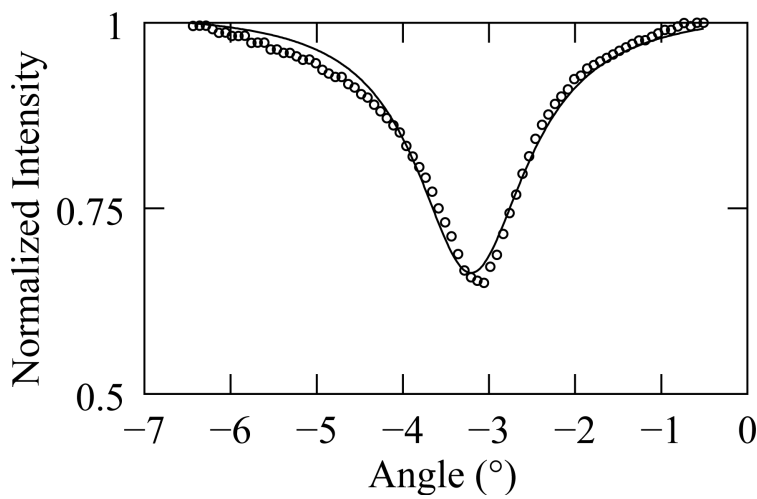

Fig. 6. Measured $(\bigcirc)$ and fitted (-) $m$-lines spectra of different ITO film on glass in TE mode.

The S1818 spectra (Fig. 4) were obtained with the Metricon setup and contain leaky modes, whereas the $\mathrm{ZnO}$ spectra (Fig. 5) were obtained with the homemade $m$-lines setup and are composed of guided modes. Both kinds of spectra are very well reproduced by the genetic algorithm; the fitness $\sigma_{m}$ is of the order of $10^{-3}$. There is also a good agreement between the refractive indices and the thicknesses given by the genetic algorithm and the classical $m$-lines analysis based on the resolution of the modal dispersion equations. The differences on the refractive index are of the order of $10^{-3}$, and the differences on the thickness are generally close to $50 \mathrm{~nm}$ but can reach $400 \mathrm{~nm}$. Let us emphasize that we do not ex- pect a perfect matching between the values given by the two methods since the classical analysis makes the assumption that there is no absorption inside the film. So, taking into account the uncertainties of both methods, the observed differences are in the acceptable range. The effective extinction factor of the resist $\mathrm{S} 1818$ was found to be close to $2.10^{-3}$ and in the case of the $\mathrm{ZnO}$ between $7.10^{-4}$ and $3.10^{-3}$ depending on the sample. These effective extinction factors of $\mathrm{ZnO}$ are in accordance with published values $[33,34]$.

The thir kind of sample, ITO layer on glass substrate, reveals another advantage of the method: it is also available when only one mode is measured, which is the case in a thin layer or in a small difference of refractive indexes between the layer and substrate. To calculate the two researched values $n$ and $d$ from the spectrum of Fig. $\underline{6}$ using the classical $m$-lines analysis, one has to use a priori information. In the present case, the thickness was assumed equal to $180 \mathrm{~nm}$ from the provider data to determine the refractive index. Such an a priori statement was not necessary with the genetic algorithm procedure. The fitted curve obtained in this way is plotted in Figs. 2(a) and 2(b) together with the measured spectrum, and a small fitness of $6.10^{-4}$ was found. There is a good agreement between the refractive index found by the classical method and the refractive index given by the genetic algorithm (GA) method. The effective extinction factor $k=0.015$ is in accordance with values commonly found for commercially available ITO [35].

\section{Conclusion}

In this paper, we have presented a method based on the transfer matrix formalism and a genetic algorithm to retrieve the optical and geometrical characteristics of a thin film from its $m$-lines spectrum and have shown its accuracy. The main interest of this method is that it not only gives the refractive index and the thickness of the film but also its effective extinction factor or optical losses. We restricted ourselves to layers with a negligible surface roughness to be able to compare the measurement of extinction factors with values found in literature; the investigation on the surface roughness effect will be the purpose of another paper. This technique does

Table 2. Characteristics of Different Thin Films Obtained with the GA and a Resolution of the Modal Dispersion Equations (MDE)

\begin{tabular}{|c|c|c|c|c|}
\hline \multirow[b]{2}{*}{ Sample } & \multicolumn{2}{|c|}{$n$} & \multicolumn{2}{|c|}{$d(\mu \mathrm{m})$} \\
\hline & GA & MDE & GA & MDE \\
\hline S1818 \#1 & $1.640 \pm 2.10^{-3}$ & $1.6401 \pm 1.10^{-4}$ & $3.407 \pm 9.10^{-3}$ & $3.80 \pm 2.10^{-2}$ \\
\hline S1818 \#2 & $1.638 \pm 2.10^{-3}$ & $1.6381 \pm 1.10^{-4}$ & $3.408 \pm 9.10^{-3}$ & $3.41 \pm 2.10^{-2}$ \\
\hline S1818 \#3 & $1.639 \pm 2.10^{-3}$ & $1.6387 \pm 1.10^{-4}$ & $3.281 \pm 9.10^{-3}$ & $3.28 \pm 2.10^{-2}$ \\
\hline $\mathrm{ZnO} \# 1$ & $1.9310 \pm 4.10^{-4}$ & $1.934 \pm 1.10^{-3}$ & $0.870 \pm 3.10^{-3}$ & $0.839 \pm 3.10^{-3}$ \\
\hline $\mathrm{ZnO} \# 2$ & $1.920 \pm 4.10^{-4}$ & $1.930 \pm 1.10^{-3}$ & $0.904 \pm 3.10^{-3}$ & $0.832 \pm 3.10^{-3}$ \\
\hline $\mathrm{ZnO} \# 3$ & $1.8600 \pm 4.10^{-4}$ & $1.861 \pm 1.10^{-3}$ & $0.587 \pm 3.10^{-3}$ & $0.554 \pm 3.10^{-3}$ \\
\hline ITO & $1.80 \pm 3.10^{-2}$ & $1.78 \pm 3.10^{-2}$ & $0.17 \pm 2.10^{-2}$ & $0.180 .18 \pm 2.10^{-2}$ \\
\hline
\end{tabular}


Table 3. Characteristics of Different Thin Films Obtained with the GA and a Resolution of the MDE

\begin{tabular}{lccc}
\hline & & $e$ & \\
$(\mu \mathrm{m})$ & $\sigma_{m}$ & $810^{-4}$ \\
Sample & $k$ & $0.105 \pm 510^{-3}$ & $310^{-4}$ \\
S1818 \#1 & $0.0028 \pm 710^{-4}$ & $0.145 \pm 510^{-3}$ & $310^{-4}$ \\
S1818 \#2 & $0.0012 \pm 710^{-4}$ & $0.152 \pm 510^{-3}$ & $210^{-3}$ \\
S1818 \#3 & $0.0021 \pm 710^{-4}$ & $3.310^{-2} \pm 210^{-3}$ & $210^{-3}$ \\
ZnO \#1 & $0.00067 \pm 610^{-5}$ & $1.710^{-3} \pm 210^{-3}$ & $110^{-3}$ \\
ZnO \#3 & $0.00071 \pm 610^{-5}$ & $2.710^{-2} \pm 210^{-3}$ & $610^{-4}$ \\
\hline ITO & $0.00260 \pm 610^{-5}$ & $0.19 \pm 0.17$ & \\
\hline
\end{tabular}

not allow us to distinguish the physical origin of optical losses between surface or volume scattering or intrinsic absorption, but it is efficient to determine which layers are more or less lossy and then to qualify films with low losses for development of advanced photonic devices. Moreover, this determination can be done in the same manner for either guided or leaky modes, and it can even be performed if only one mode is present in the spectrum. Last, since the matrix formalism allows calculating reflectivity of more complex stacks of layers, our genetic algorithm method could be easily extended to the case of bilayer or trilayer systems, which are of special interest in the fabrication of photonic components.

The authors thank John Jackson from Metricon Corporation for setup support and to Anand Srinivasan for AFM measurements.

\section{References}

1. X. J. Zhang, X. Z. Fan, J. Liao, H. T. Wang, N. B. Ming, L. Qiu, and Y. Q. Shen, "Propagation properties of a light wave in a film quasiwaveguide structure," J. Appl. Phys. 92, 56475657 (2002).

2. H. Wang, T. Aruga, and P. Ye, "Theory and properties of quasiwaveguide modes," Appl. Phys. Lett. 69, 611-613 (1996).

3. F. Abeles, "Methods for determining optical parameters of thin films," Prog. Opt. 2, 248-288 (1963).

4. J. C. Manifacier, J. Gasiot, and J. P. Fillard, "A simple method for the determination of the optical constants $n, k$ and the thickness of a weakly absorbing thin film,". J. Phys. E 9, 1002-1004 (1976).

5. J. C. Martínez-Antón, "Determination of optical parameters in general film substrate systems: a reformulation based on the concepts of envelope extremes and local magnitudes," Appl. Opt 39, 4557-4568 (2000).

6. J. M. Bennett, E. Pelletier, G. Albrand, J.-P. Borgogno, B. Lazarides, C. K. Carniglia, R. A. Schmell, T. H. Allen, T. TuttleHart, K. H. Guenther, and A. Saxer, "Comparison of the properties of titanium dioxide films prepared by various techniques," Appl. Opt. 28 3303-3317 (1989).

7. R. M. A. Azzam, "Return-path ellipsometry and a novel normal-incidence null ellipsometer (NINE)," J. Mod. Opt. 24, 1039-1049 (1977).

8. A. Bashara, Ellipsometry and Polarized Light (Academic, 1977).

9. H. G. Tompkins and W. A. McGahan, Spectroscopic Ellipsometry and Reflectometry: A User's Guide (Academic, 1999).

10. F. Flory, D. Endelema, E. Pelletier, and I. J. Hodgkinson, "Anisotropy in thin films: modeling and measurement of guided and nonguided optical properties: application to TiO 2 films," Appl. Opt 32, 5649-5659 (1993).

11. H. Rigneault, F. Flory, and S. Monneret, "Nonlinear totally reflecting prism coupler: thermomechanic effects and intensitydependent refractive index of thin films," Appl. Opt 34, 43584369 (1995).

12. P. K. Tien and R. Ulrich, "Theory of prism-film coupler and thin-film light guides," J. Opt. Soc. Am 60, 1325-1337 (1970).

13. R. Ulrich, "Theory of the prism-film coupler by plane-wave analysis," J. Opt. Soc. Am 60, 1337-1350 (1970).

14. S. Garcia-Blanco, E. Alfaro-Cid, R. M. De La Rue, and J. S. Aitchison, "Genetic algorithm-based characterization of the optical properties of flame-hydrolysis deposited silica waveguides,” J. Lightwave Technol. 22, 2801-2807 (2004).

15. J. Chilwell and I. Hodgkinson, "Thin-films field-transfer matrix theory of planar multilayer waveguides and reflection from prism-loaded waveguides," J. Opt. Soc. Am. A 1, 742753 (1984).

16. M. Born and E. Wolf, Principles of Optics (Academic, 1980).

17. Metricon Corporation, http://www.metricon.com/.

18. J. Cardin, D. Leduc, T. Schneider, C. Lupi, D. Averty, and H. W. Gundel, "Optical characterization of PZT thin films for waveguide applications," J. Eur. Ceram. Soc. 25, 2913-2916 (2005).

19. P. Yeh, Optical Waves in Layered Media (Academic, 1988).

20. A. B. H. Yedder, "Optimisation numerique et controle optimal: (applications en chimie moleculaire)," Ph.D. dissertation (Ecole Nationale des Ponts et Chaussees 2002).

21. Z. Michalewicz, Genetic Algorithms+ Data Structures $=$ Evolu tion Programs (Academic, 1996).

22. K. Deb, "Genetic algorithm in search and optimization: the technique and applications," in Proceedings of International Workshop on Soft Computing and Intelligent Systems (Academic, 1998), pp. 58-87.

23. K. Deb, Genetic Algorithms for Optimization, KanGAL Report No. 2001002, (KanGal, 2001).

24. V. S. Gordon and D. Whitley, "Serial and parallel genetic algorithms as function optimizers," in Proceedings of the Fifth International Conference on Genetic Algorithms (Academic, 1993), pp. 177-183.

25. S. W. Mahfoud, "Niching methods for genetic algorithms," $\mathrm{Ph}$. D. dissertation (University of Urbana, 1995).

26. P. J. B. Hancock, "An empirical comparison of selection methods in evolutionary algorithms," in Evolutionary Computing: AISB Workshop, Leeds, UK, (Academic, 1994), selected papers.

27. K. Deb and R. B. Agrawal, "Simulated binary crossover for continuous search space," in Complex Systems (Academic, 1995), pp. 115-148.

28. Microchem Corporation, http://www.microchem.com/products/ su_eight.htm.

29. S. Adachi, "Model dielectric constants of Si and Ge," Phys. Rev. B 38, 12966-12976 (1988).

30. Präzisions Glas \& Optik GmbH, http://www.pgo-online.com/. 
31. H. E. Bennett and J. O. Porteus, "Relation between surface roughness and specular reflectance at normal incidence," J. Opt. Soc. Am, 51, 123-129 (1961).

32. H. Davies, "The reflection of electromagnetic waves from a rough surface," in Proceedings of the Institution of Electrical Engineers (Academic, 1954), 209-214.

33. E. Dumont, B. Dugnoille, and S. Bienfait, "Simultaneous determination of the optical properties and of the structure of rfsputtered ZnO thin films," Thin Solid Films 353, 93-99 (1999).
34. M. Rebien, W. Henrion, M. Bär, and C. H. Fischer, "Optical properties of $\mathrm{ZnO}$ thin films: ion layer gas reaction compared to sputter deposition," Appl. Phys. Lett. 80, 3518-3520 (2002).

35. S. Laux, N. Kaiser, A. Zöller, R. Götzelmann, H. Lauth, and H. Bernitzki, "Room-temperature deposition of indium tin oxide thin films with plasma ion-assisted evaporation," Thin Solid Films 335, 1-5 (1998). 